\title{
Cálculo de un indicador multicriterio para la gestión de recursos humanos y tecnológicos en un servicio de hemodiálisis
}

\author{
Curchod, Miguel Ángel \\ Universidad Nacional de Córdoba (Argentina) \\ Correo electrónico: curchod@eco.unc.edu.ar \\ Alberto, Catalina Lucía \\ Universidad Nacional de Córdoba (Argentina) \\ Correo electrónico: catalina.alberto@gmail.com
}

\begin{abstract}
RESUMEN
El objetivo de este trabajo es construir un indicador compuesto, consistente y confiable, para evaluar las condiciones fisiológicas de un grupo de pacientes bajo tratamiento de hemodiálisis. Los indicadores compuestos, desarrollados a partir de indicadores individuales, tienen la ventaja de poder interpretarse rápidamente y dar a quien toma las decisiones una orientación sobre los procedimientos a seguir.

El propósito es proporcionar un indicador de gestión que ayude a optimizar el funcionamiento del servicio hospitalario para brindar a los pacientes el máximo de cuidado. Para formalizar el indicador compuesto se consideraron los laboratorios clínicos realizados a los pacientes antes del proceso de hemodiálisis.

La idea central del estudio es realizar un ranking de los pacientes sustentado en los criterios que fundamentan las condiciones fisiológicas de cada uno de ellos. El estudio se realizó en un importante hospital de la ciudad de Córdoba, Argentina.

Para resolver el problema se aplicó el método multicriterio de apoyo a las decisiones Reference Ideal Method. Se han propuesto modificaciones a la técnica originaria para que sea posible trabajar con varios intervalos ideales de referencia en un mismo criterio. La consulta a expertos para determinar los criterios de la evaluación y los pesos relativos asignados a cada uno de ellos se cumplimentó respetando las formalidades del método de indagación Delphi.
\end{abstract}

Palabras clave: Indicador de Gestión, Indicadores Clave de Performance (KPIs), Servicios hospitalarios, Análisis Multicriterio Discreto, Método RIM modificado.

Clasificación JEL: C02; C44; C65.

MSC2010: 90B50. 


\title{
Calculation of a multicriteria indicator for the management of human and technological resources in a hemodialysis service
}

\begin{abstract}
The aim of this presentation is to develop a composite indicator, which should be consistent and reliable, to evaluate the physiological conditions of a group of patients on dialysis therapy. The composite indicators are developed based on key performance indicators. They have the advantage of being able to be interpreted quickly and give to the decision makers an orientation on the procedures to be followed.

The purpose is to provide a management indicator to optimize the activities of this hospital unit, and in this way, to provide patients maximum care.

The composite indicator was built with the clinical laboratories, which are performed on patients on dialysis treatment.

The central idea of the study is to make a ranking of patients based on the criteria that support the physiological conditions of each one of them. The study was carried out in one of the major hospitals in the city of Córdoba, Argentina.

To solve the problem, we applied the multicriteria decision analysis, specifically, the Reference Ideal Method. Modifications to the traditional technique have been proposed to make it possible to work with several ideal reference intervals in the same criterion.

The referral to experts in order to define the criteria and the relative weights has been performed by application of the Delphi Method.
\end{abstract}

Keywords: Management Indicators, Key Performance Indicators (KPIs), Hospital Units, Multicriteria Decision Analysis, Modified Reference Ideal Method.

JEL classification: C02; C44; C65.

MSC2010: 90B50.






\section{Introducción.}

El objetivo del trabajo es construir un indicador multicriterio, consistente y confiable, para evaluar las condiciones fisiológicas de un grupo de pacientes que se encuentran bajo tratamiento de hemodiálisis y así poder decidir sobre la asignación de recursos humanos y tecnológicos. Los indicadores compuestos, desarrollados en base a indicadores individuales, tienen la ventaja de poder interpretarse rápidamente y dar a quien toma las decisiones una orientación inmediata sobre los procedimientos a seguir. Siguiendo a Schuschny y Soto (2009) se puede definir un indicador compuesto como "...una representación simplificada que busca resumir un concepto multidimensional en un índice simple (unidimensional) con base en un modelo conceptual subyacente" (p.13).

El origen de los indicadores compuestos se remonta a los trabajos de Burns y Mitchell en 1946 diseñados con el objetivo específico de realizar mediciones económicas. Desde ese punto de origen se han ido incrementando los campos disciplinarios para los que se han desarrollado indicadores compuestos, así como también las metodologías de construcción de dichos indicadores.

La idea central de este estudio, no es proporcionar a los médicos, especialistas en nefrología, un indicador compuesto que reemplace la interpretación detallada de los análisis clínicos individuales de los pacientes, sino otorgar a los encargados de la administración del Servicio de Hemodiálisis un instrumento adicional para realizar una gestión más eficiente y, de esta forma, brindar a los pacientes el máximo cuidado.

Téngase presente que, como señalan Nardo et al. (2008), la calidad de un indicador compuesto es consecuencia directa de los indicadores simples que lo definen. Por esta razón ellos recomiendan que los indicadores simples se seleccionen de acuerdo a su relevancia, su disponibilidad y la frecuencia con que se suministran. En este caso particular, resulta indiscutible que los indicadores simples seleccionados por los responsables de la unidad hospitalaria cumplen con los atributos señalados. Por otra parte, la construcción del indicador compuesto permite confeccionar un ranking que valora la situación fisiológica del grupo en forma global; y simultáneamente proporciona una idea sobre las condiciones fisiológicas particulares de cada uno de los pacientes. Los problemas de ranking son conocidos dentro del análisis multicriterio como problemas tipo $\gamma$ (Roy, 1985). Munda (2004) señala que el enfoque multicriterio es adecuado cuando se desea obtener un ordenamiento o ranking ya que este abordaje subsana el inconveniente de que algunas de las variables presenten objetivos contrapuestos.

Es importante destacar que existen diferentes metodologías para la construcción de indicadores compuestos. Domínguez Serrano et al. (2011) señalan distintas técnicas y explican que ellas se diferencian fundamentalmente por la forma en la que se ponderan y agregan los indicadores del sistema inicial. Una de las técnicas que estos autores citan es el enfoque propuesto por el Análisis Multicriterio. En la misma publicación los autores señalan las ventajas e inconvenientes de cada una de las metodologías por ellos abordadas.

Numerosos métodos de apoyo a las decisiones multicriterio (MCDM Multi criteria Decision Making) trabajan con valores ideales de referencia, valores máximos o mínimos para lograr el ordenamiento de las alternativas. Así lo hacen: TOPSIS (Technique for Order Preference by Similarity to Ideal Point) Yoon y Hwang (1995), VIKOR (VIseKriterijumska Optimizacija Kompromiso Resenje method) Opricovic y Tzeng (2004), MOORA (Multi- Objective Optimization on the basis of Ratio Analysis) Karel, Brauers y Zavadskas (2006); entre otros. El método seleccionado para resolver este problema es Reference Ideal Method (RIM). La diferencia fundamental que presenta este método respecto de otros métodos de MCDM es que no trabaja con un valor referencial puntual (máximo o mínimo) sino que lo hace con un conjunto de valores ideales, específicamente con un intervalo al que considera como el intervalo de referencia ideal. 
Esta característica permite que en muchas aplicaciones prácticas, el modelo resultante sea más preciso y se adapte mejor a las circunstancias reales.

El método RIM ha sido desarrollado en forma conjunta por miembros del departamento de Ingeniería de la Universidad de Holguín (Cuba) y del departamento de Inteligencia Artificial de la Universidad de Granada (España). El método fue publicado en Information Science en el año 2016 lo que lo convierte en uno de los métodos de apoyo a las decisiones multicriterio más nuevos de la bibliografía. Por este motivo podemos destacar que a la fecha no abundan los trabajos publicados aplicando esta metodología.

Dadas las características del problema analizado, se introdujeron modificaciones al método original propuesto por Cables et al. (2016) para que sea posible trabajar simultáneamente con distintos intervalos ideales de referencia en un mismo criterio según se presente algún atributo particular de los elementos del conjunto de elección. Estas incorporaciones teórico-prácticas, aportan mayor flexibilidad y potencia al método.

Por último, se mencionan algunos artículos recientes que describen la metodología de MCDM y su utilidad en el ámbito de la salud, como, por ejemplo: Zozaya et al. (2018) quienes describen los aspectos esenciales para el abordaje de problemas de salud a través de estas metodologías. Martín et al. (2005) aplican modelos de MCDM interactivos para determinar sistemas óptimos de financiación de áreas o centros sanitarios. También González (2018) pone de manifiesto la utilidad para estructurar los procesos de toma de decisiones en salud y da sustento para que estos procesos sean consistentes, transparentes y legítimos.

El trabajo se estructura de la siguiente forma. En primer lugar, se plantea el problema a resolver; posteriormente, se establece el marco teórico-conceptual explicando detalladamente el método RIM y explicitando las modificaciones propuestas. A continuación, se presenta la aplicación para el caso analizado a través de las etapas sugeridas desde la perspectiva de la racionalidad procedimental que plantean los autores. Por último, se presentan las conclusiones.

\section{El problema.}

El estudio se realizó en el servicio de hemodiálisis de un importante hospital de la ciudad de Córdoba, Argentina. La hemodiálisis es una terapia de depuración sanguínea extracorpórea cuya función es reemplazar parcialmente algunas de las funciones renales. Básicamente, el tratamiento consiste en hacer circular la sangre que se extrae de un acceso vascular (fístula, prótesis o catéter) y traspasarla a un filtro de doble compartimento llamado dializador. Por uno de los compartimentos pasa la sangre y por el otro el dializado (baño de diálisis) separados por una membrana semipermeable por la que circulan los solutos de pequeño y mediano tamaño tales como potasio, urea y creatinina e impide el paso de otros de elevado peso molecular como proteínas y células sanguíneas.

La complejidad de las prestaciones brindadas a los pacientes requiere de todos los instrumentos de gestión disponibles para optimizar el correcto funcionamiento de la unidad.

\section{EI método RIM.}

Cables et al. (2016) describen el método en las siguientes etapas o pasos.

PASO 1: determinación del contexto de trabajo especificando el rango de valores posibles para cada una de las variables y su correspondiente intervalo de referencia ideal. 
PASO 2: especificación de la matriz de respuestas de cada alternativa para cada criterio:

$\mathrm{F}(\mathrm{x})=\left[\begin{array}{cccc}\mathrm{x}_{11} & \mathrm{x}_{12} & \ldots & \mathrm{x}_{1 \mathrm{n}} \\ \mathrm{x}_{21} & \mathrm{x}_{22} & \ldots & \mathrm{x}_{2 \mathrm{n}} \\ \ldots & \ldots & \ldots & \ldots \\ \mathrm{x}_{\mathrm{m} 1} & \mathrm{x}_{\mathrm{m} 2} & \ldots & \mathrm{x}_{\mathrm{mn}}\end{array}\right]$

$x_{i j}$ representa el valor que toma la alternativa $i(i=1,2, \ldots \mathrm{m})$ en el criterio $\mathrm{j}(\mathrm{j}=1,2, \ldots \mathrm{n})$.

PASO 3: normalización de la matriz de respuestas.

$$
\overline{\mathrm{X}}=\left[\overline{\mathrm{X}_{\mathrm{ij}}}\right]
$$

Los autores proponen la normalización de la matriz de acuerdo a las siguientes funciones:

$$
\begin{aligned}
& \overline{x_{i j}} f\left(x_{i j},[A, B],[C, D]\right)=1 ; \text { si } \quad x_{i j} \in[C, D] \\
& \overline{x_{i j}} f\left(x_{i j},[A, B],[C, D]\right)=1-\frac{d_{\min }\left(x_{i j}[C, D]\right)}{[A, C]} ; \text { si } \quad x_{i j} \in[A, C] \wedge A \neq C \\
& \overline{x_{i j}} f\left(x_{i j},[A, B],[C, D]\right)=1-\frac{d_{\text {min }}\left(x_{i j}[C, D]\right)}{[D, B]} ; \text { si } \quad x_{i j} \in[D, B] \wedge D \neq B
\end{aligned}
$$

donde,

$[\mathrm{A}, \mathrm{B}]$ es el intervalo o rango de valores que puede asumir la variable.

$[C, D]$ representa el intervalo o rango de referencia ideal.

$\mathrm{x}_{\mathrm{ij}} \in[\mathrm{A}, \mathrm{B}]$.

$[\mathrm{C}, \mathrm{D}] \in[\mathrm{A}, \mathrm{B}]$.

Esta función permite asignar valores a la variable en el intervalo [0,1]. Si el valor de la variable se encuentra dentro de los límites del intervalo de referencia ideal, asumirá el valor 1. Caso contrario, a medida que se aleja de ese intervalo, el valor de la variable tenderá a cero.

PASO 4: determinación del vector de pesos asignado las ponderaciones que miden la importancia relativa para cada criterio:

$$
\mathrm{W}=\left[\begin{array}{llll}
\mathrm{w}_{1} & \mathrm{w}_{2} & \ldots & \mathrm{w}_{\mathrm{n}}
\end{array}\right]
$$


PASO 5: cálculo de la matriz normalizada y ponderada:

$\left[\overline{\mathrm{X}}^{*}\right]=[\overline{\mathrm{X}}][\mathrm{W}]$

PASO 6: determinación, para cada alternativa, de los indicadores de distancia al ideal y anti-ideal.

$$
\begin{aligned}
& I_{i}^{+}=\sqrt{\sum_{j=1}^{n}\left(\bar{x}_{i j}-w_{j}\right)^{2}} ; i=1 \ldots m \\
& I_{i}^{-}=\sqrt{\sum_{j=1}^{n}\left(\overline{x_{i j}}\right)^{2}} ; \quad i=1 \ldots m
\end{aligned}
$$

PASO 7: se calcula el indicador:

$$
\mathrm{R}_{\mathrm{i}}=\frac{\mathrm{I}_{\mathrm{i}}^{-}}{\mathrm{I}_{\mathrm{i}}^{-}+\mathrm{I}_{\mathrm{i}}^{+}} ; \mathrm{i}=1 \ldots \mathrm{m}
$$

PASO 8: formalización del ranking de preferencias de las alternativas. De acuerdo a los resultados de los cocientes calculados en [10] se determina el ordenamiento, en forma descendiente, siendo la mejor alternativa aquella que se encuentra en el primer lugar.

\subsection{Método RIM modificado.}

Para determinados tipos de problemas y para facilitar la modelización de ciertos atributos que requieren ser expresados teniendo en cuenta más de un rango de referencia de acuerdo a una $\mathrm{u}$ otra alternativa, proponemos introducir en el PASO 3 intervalos ideales de referencia diferenciados según características particulares de cada alternativa. En forma general, para el caso de un atributo con dos intervalos ideales de referencia a los que llamaremos $p$ y $q$, decimos que, todas aquellas alternativas que tengan el atributo $p$ tendrán un determinado rango e intervalo ideal de referencia y todas aquellas otras alternativas que tengan el atributo $q$ tendrán otro rango y otro intervalo ideal de referencia, siendo $p$ y $q$ mutuamente excluyentes.

Así, para todas aquellas alternativas que presenten el atributo $p$ se define un rango de valores: $[A, B]$ y un intervalo ideal de referencia $[\mathrm{C}, \mathrm{D}]$; mientras que para aquellas que tengan el atributo $q$ se establece un rango [J,K] y su intervalo ideal de referencia [L,M].

Se considera importante aclarar que de igual forma se podrían presentar más de dos atributos diferenciables, y entonces existirían tantos rangos e intervalos ideales de referencia como categorías existan dentro del conjunto de elección.

Obsérvese que los intervalos ideales de referencia son distintos y que además pueden tener diferente amplitud dado que todas aquellas alternativas que posean la característica $p$ y cuyo valor se encuentre comprendido en el intervalo ideal $[\mathrm{C}, \mathrm{D}]$ su valor normalizado será igual a 1 ; lo mismo ocurre en todas aquellas alternativas que posean la característica $q$ y cuyo valor pertenezca al intervalo ideal $[\mathrm{L}, \mathrm{M}]$. No obstante, para que las alternativas con atributo $p$ sean comparables con aquellas que tienen el atributo $q$ y cuyos valores se encuentren fuera del intervalo ideal, se 
pone la condición que se verifique que la amplitud del intervalo $[\mathrm{A}, \mathrm{C}]$ sea igual a la amplitud del intervalo $[\mathrm{J}, \mathrm{L}]$ y de igual manera, que la amplitud del intervalo $[\mathrm{D}, \mathrm{B}]$ sea igual a la amplitud del intervalo $[\mathrm{M}, \mathrm{K}]$. De esta forma se mantiene la proporcionalidad en la normalización y todas las alternativas pueden ser comparadas con el mismo razonamiento y formar parte de un mismo ranking.

En definitiva, la normalización de todas aquellas alternativas que tengan el atributo $p$ se normalizan con las funciones expresadas [3], [4] y [5] y aquellas que tengan el atributo $q$ se normalizan, en forma análoga, con funciones equivalentes pero con su rango $[\mathrm{J}, \mathrm{K}]$ y su intervalo ideal de referencia $[\mathrm{L}, \mathrm{M}]$ respetando las consideraciones expresadas respecto de las amplitudes de los intervalos.

\section{Aplicación.}

\subsection{Características del servicio de hemodiálisis.}

Con la finalidad de mejorar la gestión en la administración y organización del servicio de hemodiálisis de un hospital de la ciudad de Córdoba (Argentina), se desarrolla un indicador compuesto en base a indicadores individuales del grupo de pacientes que concurren a hemodiálisis.

El servicio de hemodiálisis de la institución trabaja con los recursos de personal que se detallan a continuación: el equipo de salud está compuesto por un médico de sala, un enfermero encargado de organizar la sesión de hemodiálisis, otros enfermeros en relación a la cantidad de pacientes (generalmente, un profesional de enfermería cada cuatro pacientes), un bioquímico y una nutricionista. También se cuenta con personal no calificado encargado de la higiene del lugar y de los servicios de refrigerio (desayuno, almuerzo, merienda o cena, de acuerdo al turno). La unidad de diálisis trabaja de lunes a sábados en 3 turnos de diarios, divididos en horarios de: mañana (de 7 a 11 horas), tarde (de 12 a 16 horas) y noche (de 17 a 21 horas). Por otra parte, el tratamiento de hemodiálisis tiene las siguientes particularidades: los pacientes concurren 3 veces por semana en el turno en que fueron asignados, el tiempo de cada sesión es, en general, de 4 horas, pudiendo excepcionalmente, variar de acuerdo al grado de funcionamiento renal, ganancia de peso entre sesiones, $\mathrm{y}$ al estado físico y condiciones de salud de paciente.

El trabajo se realizó sobre la totalidad de los pacientes del turno noche que concurren los días lunes, miércoles y viernes. El grupo de análisis tiene las siguientes características: a) el total de pacientes bajo estudio son $15, \mathrm{~b}$ ) el paciente más joven del grupo tiene 16 años, c) el mayor de ellos tiene 76 años, d) la amplitud del intervalo es de 60 años, e) la edad promedio es 54 años, f) la cantidad de pacientes de sexo masculino es $11, \mathrm{~g}$ ) la cantidad de pacientes de sexo femenino es $4, \mathrm{~h}$ ) los indicadores individuales (variables o criterios de evaluación) que se tomaron en cuenta para el desarrollo del indicador compuesto son los análisis clínicos que se realizan a los pacientes antes del proceso de diálisis.

\subsection{Estructuración y resolución del problema.}

Para la resolución del problema se consideran los pasos detallados en el apartado 3.

PASO 1: Determinación del contexto de trabajo.

En este paso se identifican de las alternativas, se definen las variables, su rango y su intervalo ideal de referencia. 
Alternativas: el conjunto de alternativas a evaluar está constituido por los 15 pacientes del servicio de hemodiálisis (turno noche).

$$
\text { En forma comprensiva: }\left[\text { Pcte }_{\mathrm{i}} \in \mathrm{A} ; \mathrm{i} \in I=\{\mathrm{i} / \mathrm{i} \in N \wedge 1 \leq \mathrm{n} \leq 15\}\right]
$$

siendo:

Pcte $_{i}=$ Paciente $i$

$\mathrm{A}=$ Conjunto de Alternativas

Por razones de privacidad de los pacientes, no se publica su identificación. No obstante, para la interpretación de los datos y de los resultados, se puede aclarar, sin quebrantar las normas éticas señaladas, que se los ha referido con un código cuyos últimos tres dígitos se corresponden con el sexo $(\mathrm{H}=$ hombre; $\mathrm{M}=$ mujer $)$ y los números indican la edad del paciente.

Definición de variables: Las variables fueron seleccionadas por los médicos especialistas en nefrología y coinciden con los análisis de laboratorio que habitualmente se realizan a los pacientes antes de comenzar la sesión de hemodiálisis. Estas son: 1. Hemoglobina (HB); 2. Volumen Corpuscular Medio (VCM); 3. Hematocrito (HTO); 4. Albúmina (ALB); 5. Sodio (NA); 6. Potasio (K); 7. Fósforo (P); 8. Creatina (CREAT.); 9. Urea (UREA); 10. Glutamato Oxalacetato Transaminasa (GOT); 11. Glutamato Piruvato Transaminasa (GPT); 12. Fosfatasa Alcalina (FOSF. ALC.); 13. Parathormona Molécula Intacta (PTH).

Los especialistas consultados explicaron que las variables 1, 2 y 3 son los componentes más importantes del hemograma del paciente y que, por lo tanto, son las que proporcionan información de calidad respecto del sistema cardiovascular. Las variables 4 a 9 se corresponden con la química clínica. Las variables 10, 11 y 12 se refieren a la función hepática y, finalmente, el indicador 13 atañe al metabolismo mineral. Además, señalaron que es fundamental que todos estos valores se interpreten bajo la óptica de pacientes bajo terapia de hemodiálisis.

La mayoría de los laboratorios utilizados como indicadores individuales para desarrollar el indicador compuesto, no pueden ser definidos como criterios de máximo o de mínimo.

Estas variables tienen cotas inferiores y superiores dentro de las que el valor de la variable se considera normal. El superar la cota máxima significa una alternación fisiológica del paciente que es reflejada por el análisis considerado. De la misma forma, no alcanzar la cota mínima también representa un desvío de la normalidad deseada.

En el ANEXO se detallan los Cuadros 1 al 5 que muestran los resultados obtenidos en los diferentes pasos de la aplicación.

En el Cuadro 1 se detallan los indicadores individuales mencionados, su rango de valores posibles y los valores mínimos y máximos entre los que los marcadores se consideran normales. Estos valores normales constituyen para nuestro trabajo los intervalos ideales de cada indicador.

PASO 2. Con los resultados de los análisis de laboratorios realizados a los pacientes antes de la sesión de hemodiálisis se construyó la matriz de respuesta que se detalla en el Cuadro 2.

PASO 3. Para calcular la matriz normalizada, se debe tener en cuenta que en este problema, los valores de los intervalos ideales de referencia, en los análisis de: Hemoglobina, Hematocrito y Creatinina varían según el paciente sea hombre o mujer (Cuadro 1); por lo tanto, se trabajó con la modificación del método RIM propuesta en el apartado 3.1. De esta manera, dentro de los elementos del conjunto de alternativas, se diferencian los intervalos ideales de referencia según el atributo: "sexo" y por el valor cualitativo que el atributo puede asumir: "Hombre" o "Mujer". 
La matriz normalizada obtenida mediante este procedimiento se muestra en el Cuadro 3. Como se mencionó anteriormente, este procedimiento podría utilizarse si el problema presentara más de dos atributos diferenciables (como, por ejemplo: niños y adultos, o niños y adultos hombres y adultos mujeres, etc.), entonces existirían tantos intervalos ideales de referencia como categorías existan dentro del conjunto de elección.

PASO 4. Consiste en determinar el vector de pesos relativos asignados a cada una de las variables. Esta etapa se conformó a través de entrevistas personales con los médicos a cargo del departamento de hemodiálisis. Es importante destacar que el panel de expertos de asesoramiento que colaboró en esta aplicación se constituyó considerando el conocimiento que los nefrólogos consultados tienen sobre la institución y sobre las tareas profesionales específicas que desempeñan.

La indagación se formalizó observando rigurosamente los requisitos procedimentales del método Delphi; es decir: a) anonimato, b) cuestionarios sucesivos con retroalimentación controlada, c) independencia de los expertos y d) análisis de las respuestas del grupo.

Se indagó sobre la relevancia que tenía cada una de las variables para evaluar las condiciones de salud de los pacientes. Debido a que los criterios definidos para evaluar las condiciones fisiológicas de los pacientes son numerosos resultó muy difícil para el panel de expertos ponderar los indicadores individuales y llegar a un consenso.

Las discrepancias que se presentaron estaban fundamentadas tanto por las diferentes experiencias profesionales de los miembros del panel, como así también, por las escuelas científicas en las que cada uno de ellos se enrola para ejercer su profesión.

Para simplificar y facilitar la tarea de determinar la importancia relativa de los criterios, se solicitó a los especialistas que realizaran un ranking de importancia de los análisis de laboratorio que habitualmente se prescriben antes de comenzar la terapia. De esta forma, se logró acuerdo para jerarquizar los indicadores individuales y consolidar un ranking en función de la información que cada uno de los análisis brinda respecto de las condiciones fisiológicas de los pacientes y de los riesgos que suponen resultados alejados del intervalo ideal.

Con el listado de la posición de importancia realizado se aplicó la siguiente fórmula correspondiente al método de asignación directa:

$$
\mathrm{w}_{j}=\frac{\left(n-P_{j}+1\right)}{\sum_{i=1}^{n}\left(n-P_{j}+1\right)}
$$

siendo:

$\mathrm{n}=$ cantidad de criterios.

$\mathrm{P}_{\mathrm{j}}=$ posición que ocupa el criterio $j$ en el conjunto de criterios de acuerdo a su importancia.

La fórmula [11] permite, en el numerador, valorar la importancia relativa del criterio de acuerdo a la posición que ocupa dentro del conjunto y normalizarla a través del denominador.

Este procedimiento se estima pertinente teniendo en cuenta el número significativo de variables definidas, y las consideraciones teóricas que realiza el $M C D A$ respecto de las propiedades que deben cumplir los criterios (exhaustivos, coherentes y no redundantes). 
El posicionamiento de importancia de las variables logrado se ha respetado en la construcción de los Cuadros 1 al 4 y los pesos asignados se muestran en el Cuadro 5.

PASO 5. Construcción de la matriz normalizada y ponderada por los pesos relativos asignados a cada criterio, los resultados de esta etapa se detallan en el Cuadro 4.

PASO 6. Determinación para cada alternativa de los indicadores de distancia ideal "I+" se calculó de acuerdo a la fórmula [8] y los indicadores de distancia anti ideal "I-" de acuerdo a la fórmula [9].

PASO 7. Se calculó el indicador detallado en [10].

PASO 8. Finalmente, se pudo determinar el ranking de los pacientes que se muestra en el Cuadro 5.

\section{Conclusiones.}

Los resultados obtenidos de la aplicación fueron analizados en forma conjunta con el jefe de turno de la Unidad de Hemodiálisis teniendo en cuenta su experticia y la anamnesis de cada uno de los pacientes, de esa forma se pudo concluir que el ranking logrado tenía plena sustentabilidad. El primer lugar lo ocupa BA H22 con un indicador de 0,8675 . El paciente es una persona joven, con buena nutrición, quien aceptó sin mayores dificultades su tratamiento, prolijo en las asistencias a la terapia de diálisis, con buena tolerancia al tratamiento y ganancia de peso mínima entre sesiones (de 1.000 a 1.500 gramos). La antigüedad en el tratamiento de diálisis es de 4 meses aproximadamente. En contraposición el último lugar lo ocupa RJ H40 con un indicador de 0,5145. Este paciente es un adulto joven con buena nutrición, pero con complicaciones en su salud debido a la no aceptación de su enfermedad. Con ganancias de peso entre sesiones de diálisis de $4.500 \mathrm{a}$ 6.000 gramos aproximadamente. La antigüedad en el tratamiento es de 6 años. El segundo lugar lo ocupó una paciente de 65 años con un indicador de 0.7972. El paciente más joven del grupo (16 años) ocupa la posición número 13. Considerando estas posiciones se pudo inferir que, si bien la edad del paciente no fue incorporada entre los indicadores individuales para desarrollar el indicador compuesto, se pudo verificar, como señalaron a priori los expertos, que la edad no es una variable determinante en las condiciones fisiológicas del enfermo bajo tratamiento de hemodiálisis. También, de acuerdo a la opinión de los especialistas y haciendo un análisis similar al anterior se infiere que el sexo tampoco es una variable determinante de las condiciones fisiológicas de los pacientes. Como medida de intervención se indicó que el rango del indicador se divida en intervalos de: alarma, atención o conformidad. (Una primera aproximación dada por los expertos fue entre: $0-0,20 ; 0,20-0,50$; más de 0,50 ). Resultando los más comprometidos aquellos pacientes con indicadores en el intervalo más bajo. Por la razón anterior, se recomendó un seguimiento más minucioso de aquellos pacientes que ocupan las últimas posiciones del ranking. A ellos se les debería asignar el personal con mayor experiencia y los recursos tecnológicos más eficaces. En este caso particular las medidas de estadística descriptiva de la población analizada fueron: todos los pacientes presentan buenas mediciones del indicador compuesto, superiores en todos los casos a 0,50 (concretamente: 0,5145). El rango de amplitud del indicador es 0,3530 . El valor promedio es 0,7027 y la desviación estándar es 0,09 . Por otra parte, es relevante tener en cuenta que los análisis de laboratorios son mediciones dinámicas, que varían constantemente, estos cambios no tienen una dispersión significativa entre sesiones consecutivas; sin embargo, es recomendable que el indicador compuesto se determine al menos mensualmente, con la finalidad de detectar alguna variación significativa para poder prevenir un punto de giro relevante en las condiciones fisiológicas del paciente.

Respecto de la metodología multicriterio empleada para la determinación del indicador compuesto se puede concluir que resulta adecuada. El método RIM y las modificaciones 
incorporadas permitieron modelizar en forma precisa el problema bajo análisis. Además, se puede alegar que el desarrollo de indicadores compuestos es relevante a la hora de tomar decisiones proporcionando un instrumento que presenta la información en forma integrada, concisa y representativa.

\section{Referencias}

Burns, A.F., \& Mitchell, W.C. (1946). Measuring business cycles. Studies in business cycles. USA: National Bureau of Economic Research. https://www.jstor.org/stable/1826221

Cables, E., Lamata, M. T., \& Verdegay, J. L. (2016). RIM reference ideal method in multicrtiteria $\begin{array}{lllll}\text { decision making. } & \text { Information }\end{array}$ https://www.sciencedirect.com/science/article/abs/pii/S0020025515009007

Domínguez, M., Blancas, F.J., Guerrero, F.M., \& González, M. (2011). Una revisión crítica para la construcción de indicadores sintéticos. Métodos Cuantitativos para la Economía y la Empresa 11, 41-70. https://www.upo.es/revistas/index.php/RevMetCuant/article/view/2094

González, D. (11 de octubre de 2018). www.neuroeconomics.com/analisis-de-decisionmulticriterio-mcda/. Recuperado el 15 de abril de 2020, de www.neuroeconomics.com/analisis-de-decision-multicriterio-mcda/

Karel, W., Brauers, W., \& Zavadskas, E. (2006). The MOORA method and its applications to privatization in a transition economic. Control and Cybernetics 35, 2. https://citeseerx.ist.psu.edu/viewdoc/download?doi=10.1.1.379.8910\&rep=rep1\&type=pdf

Martín, J., Puerto, M., Caballero, R., \& Luque, M. (January de 2005). Financiación de Hospitales y Asignación de Recursos mediante Técnicas Multicriterio Interactivas. Recuperado el 25 de abril de 2020, de https://www.researchgate.net/publication/229023093_Financiacion_de_hospitales_y_asignac ion_de_recursos_mediante_tecnicas_multicriterio_interactivas:

http://www.uib.es/congres/ecopub/papers/sanidad/Martin-Puerto-Caballero-Luque.pdf.

Munda, G. (2004). Métodos y Procesos Multicriterio para la Evaluación Social de las Políticas Públicas. Iberoamericana de Economía Ecológica, 1, 31-45. https://raco.cat/index.php/Revibec/article/view/38279 [Consulta: 24-08-2021

Nardo, M., Saisana, M., Saltelli, A., Tarantla, S., Hoffman, A., \& Giovannnini, E. (2008). Handbook on constructing composite indicators: methodology user guide. Paris: OECD European Union. https://unstats.un.org/unsd/EconStatKB/KnowledgebaseArticle10366.aspx

Opricovic, S., \& Hshiung, G. (2004). Compromise Solution by MCDM methods: a comparative analysis of Vikor and Topsis. European Journal of Orational Research, 156, 445-455. https://www.sciencedirect.com/science/article/abs/pii/S0377221703000201

Roy, B. (1985). Méthodology multicritere d'aide a la decision. Paris: Económica.

Schuschny, A., \& Soto, H. (2009). Guía Metodológica. Diseño de indicadores compuestos de desarrollo sostenible. Santiago de Chile: Comisión Economica para América Latina y el Caribe (CEPAL). https://www.bivica.org/file/view/id/2485

Yonn, K., \& Hwang, C. L. (1995). Multiple Attribute Decision Making: An Introduction. California, USA: Sage. 
Zozaya, N., Oliva, J., Hidalgo, Á., \& García, A. (2018). El Análisis de Decisión Multi-Criterio en el ámbito sanitario: utilidad y limitaciones para la toma de decisiones. Madrid - España: Fundación Weber. https://weber.org.es/publicacion/el-analisis-de-decision-multi-criterio-enel-ambito-sanitario-utilidad-y-limitaciones-en-la-toma-de-decisiones/ 
ANEXO

Cuadro 1. Entorno de Trabajo.

\begin{tabular}{|c|c|c|c|c|c|c|c|c|c|c|c|c|c|}
\hline COLUMNA & 1 & 2 & 3 & 4 & 5 & 6 & 7 & 8 & 9 & 10 & 11 & 12 & 13 \\
\hline $\begin{array}{l}\text { VARIABLE / } \\
\text { CRITERIO }\end{array}$ & $\mathrm{K}$ & HB & ALB & $\mathrm{P}$ & UREA & NA & GOT & GPT & HTO & CREAT. & $\begin{array}{l}\text { FOSF. } \\
\text { ALC. }\end{array}$ & PTH & VCM \\
\hline RANGO A & 3 & 6 & 3 & 2,5 & 5 & 125 & 5 & 5 & 18 & 0,25 & 120 & 10 & 70 \\
\hline RANGO B & 7 & 16 & 5,5 & 8 & 255 & 150 & 45 & 80 & 55 & 16 & 3850 & 2200 & 100 \\
\hline C IDEAL MIN & 3,5 & 13 & 3,5 & 2,7 & 15 & 135 & 10 & 7 & 43 & 0,5 & 91 & 50 & 80 \\
\hline D IDEAL MAX & 5 & 15 & 5 & 4,5 & 50 & 147 & 40 & 41 & 50 & 1,3 & 258 & 325 & 95 \\
\hline RANGO J & 3 & 5 & 3 & 2,5 & 5 & 125 & 5 & 5 & 11 & 0,25 & 120 & 10 & 70 \\
\hline RANGO K & 7 & 15 & 5,5 & 8 & 255 & 150 & 45 & 80 & 49 & 15,8 & 3850 & 2200 & 100 \\
\hline L IDEAL MIN & 3,5 & 12 & 3,5 & 2,7 & 15 & 135 & 10 & 7 & 36 & 0,5 & 91 & 50 & 80 \\
\hline M IDEAL MAX & 5 & 14 & 5 & 4,5 & 50 & 147 & 40 & 41 & 44 & 1,1 & 258 & 325 & 95 \\
\hline
\end{tabular}

Fuente: Elaboración propia. 
Cuadro 2. Matriz de Respuestas.

\begin{tabular}{|c|c|c|c|c|c|c|c|c|c|c|c|c|c|}
\hline PACIENTE & $\mathbf{K}$ & $\mathbf{H b}$ & ALB & $\mathbf{P}$ & UREA & NA & GOT & GPT & HTO & CREAT. & $\begin{array}{l}\text { FOSF. } \\
\text { ALC. }\end{array}$ & PTH & VCM \\
\hline AJ H62 & 4,70 & 8,50 & 3,10 & 4,90 & 151,00 & 138,00 & 14,00 & 17,00 & 26,20 & 11,22 & 282,00 & 320,00 & 82,60 \\
\hline AO H70 & 4,00 & 10,50 & 3,60 & 4,00 & 189,00 & 139,00 & 12,00 & 14,00 & 32,00 & 8,59 & 198,00 & 183,00 & 93,30 \\
\hline AE M 66 & 4,40 & 10,30 & 3,70 & 5,70 & 162,00 & 137,00 & 20,00 & 22,00 & 32,40 & 9,06 & 464,00 & 504,00 & 86,20 \\
\hline BA H22 & 3,70 & 13,40 & 4,20 & 3,20 & 134,00 & 140,00 & 11,00 & 18,00 & 41,90 & 8,39 & 404,00 & 592,00 & 89,50 \\
\hline BA M65 & 4,00 & 10,20 & 3,70 & 6,00 & 107,00 & 137,00 & 12,00 & 15,00 & 31,90 & 7,67 & 787,00 & $1.708,00$ & 89,10 \\
\hline CJ H67 & 3,10 & 12,80 & 3,90 & 3,80 & 79,00 & 137,00 & 10,00 & 16,00 & 37,50 & 4,13 & 186,00 & 134,00 & 84,80 \\
\hline CH M36 & 4,90 & 10,10 & 4,00 & 3,10 & 182,00 & 134,00 & 12,00 & 18,00 & 29,60 & 13,60 & 179,00 & 406,00 & 88,90 \\
\hline CA H56 & 5,30 & 11,10 & 4,40 & 7,20 & 164,00 & 141,00 & 7,00 & 13,00 & 34,20 & 11,50 & 707,00 & $1.760,00$ & 98,60 \\
\hline ME H16 & 3,70 & 7,40 & 3,40 & 3,70 & 145,00 & 138,00 & 15,00 & 14,00 & 21,40 & 10,93 & 3807,00 & $1.556,00$ & 88,40 \\
\hline OD H73 & 5,30 & 8,90 & 3,60 & 3,20 & 103,00 & 140,00 & 14,00 & 19,00 & 29,10 & 4,32 & 126,00 & 103,00 & 85,30 \\
\hline PC H47 & 5,00 & 12,50 & 4,00 & 6,40 & 199,00 & 136,00 & 9,00 & 17,00 & 37,10 & 15,47 & 240,00 & 745,00 & 91,20 \\
\hline PC H45 & 4,50 & 13,80 & 3,70 & 6,00 & 153,00 & 139,00 & 10,00 & 35,00 & 42,40 & 11,74 & 322,00 & 489,00 & 91,80 \\
\hline RJ H40 & 6,90 & 9,60 & 3,80 & 6,50 & 214,00 & 134,00 & 14,00 & 16,00 & 29,50 & 14,15 & 736,00 & $2.103,00$ & 90,20 \\
\hline SJ H76 & 5,00 & 12,10 & 3,90 & 8,00 & 251,00 & 138,00 & 12,00 & 19,00 & 35,60 & 8,32 & 320,00 & 688,00 & 86,80 \\
\hline SJ M64 & 5,10 & 6,90 & 3,50 & 5,60 & 202,00 & 128,00 & 38,00 & 70,00 & 19,70 & 7,59 & 355,00 & 397,00 & 92,50 \\
\hline MINIMO & 3,10 & 6,90 & 3,10 & 3,10 & 79,00 & 128,00 & 7,00 & 13,00 & 19,70 & 4,13 & 126,00 & 103,00 & 82,60 \\
\hline MAXIMO & 6,90 & 13,80 & 4,40 & 8,00 & 251,00 & 141,00 & 38,00 & 70,00 & 42,40 & 15,47 & 3807,00 & 2103,00 & 98,60 \\
\hline
\end{tabular}

Fuente: Elaboración propia. 
Cuadro 3. Matriz Normalizada.

\begin{tabular}{|c|c|c|c|c|c|c|c|c|c|c|c|c|c|}
\hline $\begin{array}{l}\text { COD. } \\
\text { PACIEN. }\end{array}$ & $\mathbf{K}$ & $\mathbf{H b}$ & ALB & $\mathbf{P}$ & UREA & NA & GOT & GPT & HTO & CREAT. & $\begin{array}{r}\text { FOSF. } \\
\text { ALC. }\end{array}$ & PTH & VCM \\
\hline AJ H62 & 1,00 & 0,36 & 0,20 & 0,89 & 0,51 & 1,00 & 1,00 & 1,00 & 0,33 & 0,33 & 0,99 & 1,00 & 1,00 \\
\hline AO H70 & 1,00 & 0,64 & 1,00 & 1,00 & 0,32 & 1,00 & 1,00 & 1,00 & 0,56 & 0,50 & 1,00 & 1,00 & 1,00 \\
\hline AE M 66 & 1,00 & 0,76 & 1,00 & 0,66 & 0,45 & 1,00 & 1,00 & 1,00 & 0,86 & 0,46 & 0,94 & 0,90 & 1,00 \\
\hline BA H22 & 1,00 & 1,00 & 1,00 & 1,00 & 0,59 & 1,00 & 1,00 & 1,00 & 0,96 & 0,52 & 0,96 & 0,86 & 1,00 \\
\hline BA M65 & 1,00 & 0,74 & 1,00 & 0,57 & 0,72 & 1,00 & 1,00 & 1,00 & 0,84 & 0,55 & 0,85 & 0,26 & 1,00 \\
\hline CJ H67 & 0,20 & 0,97 & 1,00 & 1,00 & 0,86 & 1,00 & 1,00 & 1,00 & 0,78 & 0,81 & 1,00 & 1,00 & 1,00 \\
\hline CH M36 & 1,00 & 0,73 & 1,00 & 1,00 & 0,36 & 1,00 & 1,00 & 1,00 & 0,74 & 0,15 & 1,00 & 0,96 & 1,00 \\
\hline CA H56 & 0,85 & 0,73 & 1,00 & 0,23 & 0,44 & 1,00 & 0,40 & 1,00 & 0,65 & 0,31 & 0,88 & 0,23 & 0,28 \\
\hline ME H16 & 1,00 & 0,20 & 0,40 & 1,00 & 0,54 & 1,00 & 1,00 & 1,00 & 0,14 & 0,34 & 0,01 & 0,34 & 1,00 \\
\hline OD H73 & 0,85 & 0,41 & 1,00 & 1,00 & 0,74 & 1,00 & 1,00 & 1,00 & 0,44 & 0,79 & 1,00 & 1,00 & 1,00 \\
\hline PC H47 & 1,00 & 0,93 & 1,00 & 0,46 & 0,27 & 1,00 & 0,80 & 1,00 & 0,76 & 0,04 & 1,00 & 0,78 & 1,00 \\
\hline PC H45 & 1,00 & 1,00 & 1,00 & 0,57 & 0,50 & 1,00 & 1,00 & 1,00 & 0,98 & 0,29 & 0,98 & 0,91 & 1,00 \\
\hline RJ H40 & 0,05 & 0,51 & 1,00 & 0,43 & 0,20 & 0,90 & 1,00 & 1,00 & 0,46 & 0,13 & 0,87 & 0,05 & 1,00 \\
\hline SJ H76 & 1,00 & 0,87 & 1,00 & 0,00 & 0,02 & 1,00 & 1,00 & 1,00 & 0,70 & 0,52 & 0,98 & 0,81 & 1,00 \\
\hline SJ M64 & 0,95 & 0,27 & 1,00 & 0,69 & 0,01 & 0,30 & 1,00 & 0,26 & 0,35 & 0,56 & 0,97 & 0,96 & 1,00 \\
\hline
\end{tabular}

Fuente: Elaboración propia. 
Cuadro 4. Matriz Normalizada y Ponderada.

\begin{tabular}{llllllllllllll}
\hline PESOS & 0,1429 & 0,1310 & 0,1209 & 0,1099 & 0,0989 & 0,0879 & 0,0769 & 0,0659 & 0,0549 & 0,0440 & 0,0330 & 0,0220 & 0,0118 \\
\hline $\begin{array}{l}\text { COD. } \\
\text { PACIEN. }\end{array}$ & K & Hb & ALB & P & UREA & NA & GOT & GPT & HTO & CREAT. & FOSF. & PTH & VCM \\
AJ H62 & 0,1429 & 0,0468 & 0,0242 & 0,0973 & 0,0502 & 0,0879 & 0,0769 & 0,0659 & 0,0180 & 0,0143 & 0,0328 & 0,0220 & 0,0118 \\
AO H70 & 0,1429 & 0,0842 & 0,1209 & 0,1099 & 0,0318 & 0,0879 & 0,0769 & 0,0659 & 0,0307 & 0,0222 & 0,0330 & 0,0220 & 0,0118 \\
AE M 66 & 0,1429 & 0,0992 & 0,1209 & 0,0722 & 0,0449 & 0,0879 & 0,0769 & 0,0659 & 0,0470 & 0,0202 & 0,0311 & 0,0199 & 0,0118 \\
BA H22 & 0,1429 & 0,1310 & 0,1209 & 0,1099 & 0,0584 & 0,0879 & 0,0769 & 0,0659 & 0,0525 & 0,0228 & 0,0317 & 0,0189 & 0,0118 \\
BA M65 & 0,1429 & 0,0973 & 0,1209 & 0,0628 & 0,0714 & 0,0879 & 0,0769 & 0,0659 & 0,0459 & 0,0243 & 0,0281 & 0,0058 & 0,0118 \\
CJ H67 & 0,0286 & 0,1273 & 0,1209 & 0,1099 & 0,0849 & 0,0879 & 0,0769 & 0,0659 & 0,0428 & 0,0355 & 0,0330 & 0,0220 & 0,0118 \\
CH M36 & 0,1429 & 0,0954 & 0,1209 & 0,1099 & 0,0352 & 0,0879 & 0,0769 & 0,0659 & 0,0408 & 0,0066 & 0,0330 & 0,0210 & 0,0118 \\
CA H56 & 0,1215 & 0,0954 & 0,1209 & 0,0251 & 0,0439 & 0,0879 & 0,0308 & 0,0659 & 0,0356 & 0,0135 & 0,0289 & 0,0052 & 0,0033 \\
ME H16 & 0,1429 & 0,0262 & 0,0484 & 0,1099 & 0,0531 & 0,0879 & 0,0769 & 0,0659 & 0,0075 & 0,0152 & 0,0004 & 0,0076 & 0,0118 \\
OD H73 & 0,1215 & 0,0543 & 0,1209 & 0,1099 & 0,0733 & 0,0879 & 0,0769 & 0,0659 & 0,0244 & 0,0350 & 0,0330 & 0,0220 & 0,0118 \\
PC H47 & 0,1429 & 0,1216 & 0,1209 & 0,0502 & 0,0270 & 0,0879 & 0,0615 & 0,0659 & 0,0419 & 0,0016 & 0,0330 & 0,0171 & 0,0118 \\
PC H45 & 0,1429 & 0,1310 & 0,1209 & 0,0628 & 0,0492 & 0,0879 & 0,0769 & 0,0659 & 0,0536 & 0,0128 & 0,0324 & 0,0201 & 0,0118 \\
RJ H40 & 0,0071 & 0,0674 & 0,1209 & 0,0471 & 0,0198 & 0,0791 & 0,0769 & 0,0659 & 0,0253 & 0,0055 & 0,0286 & 0,0011 & 0,0118 \\
SJ H76 & 0,1429 & 0,1142 & 0,1209 & 0,0000 & 0,0019 & 0,0879 & 0,0769 & 0,0659 & 0,0386 & 0,0230 & 0,0324 & 0,0177 & 0,0118 \\
SJ M64 & 0,1358 & 0,0356 & 0,1209 & 0,0754 & 0,0014 & 0,0264 & 0,0769 & 0,0169 & 0,0191 & 0,0246 & 0,0321 & 0,0212 & 0,0118 \\
\hline
\end{tabular}

Fuente: Elaboración propia. 
Cuadro 5. Ranking.

\begin{tabular}{ccc}
\hline RANKING & PACIENTE & VALOR \\
\hline 1 & BA H22 & 0,8675 \\
2 & BA M65 & 0,7972 \\
3 & PC H45 & 0,7910 \\
4 & AE M 66 & 0,7788 \\
5 & CH M36 & 0,7715 \\
6 & AO H70 & 0,7584 \\
7 & OD H73 & 0,7489 \\
8 & PC H47 & 0,7189 \\
9 & CJ H67 & 0,7003 \\
10 & CA H56 & 0,6543 \\
11 & SJ H76 & 0,6376 \\
12 & AJ H62 & 0,6177 \\
13 & ME H16 & 0,6127 \\
14 & SJ M64 & 0,5720 \\
15 & RJ H40 & 0,5145 \\
\hline
\end{tabular}

Fuente: Elaboración propia. 